\title{
Principales Problemas de los Profesores Principiantes en la Enseñanza Universitaria
}

\author{
Irene Fondón, María J. Madero y Auxiliadora Sarmiento \\ Universidad de Sevilla, Departamento de Teoría de la Señal y Comunicaciones, \\ Escuela Técnica Superior de Ingenieros, C/ Camino de los Descubrimientos s/n \\ C.P. 41092 Sevilla-España (e-mail: irenef@us.es, mjmadero@us.es, sarmiento@us.es )
}

\section{Resumen}

Se presentan y discuten algunas reflexiones sobre los principales problemas que los profesores principiantes encuentran en la enseñanza universitaria. Dichas dificultades se clasifican y analizan en tres ámbitos: el de la enseñanza, el de las relaciones interpersonales y el de la gestión o el contexto institucional. Se resalta la importancia de una adecuada formación pedagógica por parte del docente novel y el papel de la acción tutorial. Se revisa también los retos que suponen para el profesor principiante la actual reforma del modelo universitario español en el marco del Espacio Europeo de Educación Superior y el conflicto investigación-docencia. Esto porque la actividad investigadora no sólo es indispensable para la continua evolución científica del profesor universitario, sino que también depende de ella su continuidad en la carrera docente. Dicha actividad es a menudo difícil de compatibilizar con la puramente docente, especialmente para el docente principiante.

Palabras clave: enseñanza universitaria, profesores principiantes, investigación y docencia

\section{Main Problems of Novice Instructors in University Teaching}

\begin{abstract}
Some reflections on the main problems that novice university instructors face in higher education are presented and discussed. Such difficulties are classified and analyzed in three aspects: that of teaching, that of interpersonal relationships and that of management or institutional context. The importance of an adequate pedagogical training of the novice teacher and the role of the tutorial action are emphasized. The challenges that the novice instructor must face in the present reform of the Spanish university model according to the European Space for Higher Education and the research-teaching conflict are reviewed. This because research activity is not only indispensable for the constant scientific evolution of the university professor, but it is also an aspect that may guarantee continuity of the professor in the university career. Such activity is usually difficult to combine with the purely educational, especially for novice instructors.
\end{abstract}




\section{INTRODUCCIÓN}

En las últimas décadas, se está prestando especial atención al estudio de los problemas con los que se encuentran los profesores principiantes en el inicio de su carrera docente en el ámbito universitario, como prueban múltiples estudios e investigaciones, (Mignorance-Díaz et al. 1993; Cruz et al., 1999; Bozu, 2009). Por regla general, estos problemas se refieren fundamentalmente a aspectos relacionados con la docencia, recursos, investigación y relación con los compañeros, (Dunkin, 1990). La tarea fundamental del profesor principante durante sus primeros años de docencia debe ser el aprendizaje, dirigido a enfocar su pensamiento y sus comportamientos hacia las demandas de la enseñanza, (Howwey, 1988). Sin embargo, la Universidad suele dejar en manos del profesor la labor de formación pedagógica, a pesar de que prestar atención al período de iniciación es una inversión rentable, tanto desde el punto de vista de la permanencia del profesorado como de la responsabilidad de asegurar una enseñanza de más calidad, como profesionales mejor preparados, (Marcelo, 1999).

La realidad es que el nivel académico de los profesores en la Universidad está garantizado dado el proceso de inserción en la carrera docente universitaria, pero esto mismo no ocurre en relación con la preparación para la función docente. Sin la necesaria formación específica para el desempeño docente, el profesor principiante puede quedar desprotegido y sentirse perdido ante el nuevo reto que afronta, dado que en esta etapa el profesorado se enfrenta a las diferencias entre los ideales y la realidad, (Veenam, 1984). La falta de experiencia suele suplirse por un gran interés y motivación que hacen que el propio docente busque la forma de mejorar su labor docente por sí mismo. Sin embargo, existe el peligro de no encontrar los mecanismos adecuados o caer en la desmotivación si la respuesta del alumnado no es la deseada. Afortunadamente, en la mayoría de las universidades, existen los denominados programas de formación de profesorado novel. Estos programas, de participación voluntaria, proporcionan el apoyo y asesoramiento didáctico y pedagógico necesarios para afrontar el inicio de la carrera docente. Para ello se forman equipos docentes constituidos por profesores noveles y uno o varios profesores mentores, que acreditan una amplia experiencia como docentes. Estos programas son, además, especialmente útiles para el estudio en profundidad de la problemática de los profesores noveles, (Colén et al., 2000; Sánchez-Moreno y Mayor-Ruiz, 2006).

El profesorado debe desempeñar tareas docentes, investigadoras y de gestión. Sin embargo, en los procesos de promoción dentro de la universidad se valora más la tarea investigadora frente a las tareas docente y de gestión. En el profesorado principiante, esto se traduce en el denominado conflicto investigación-docencia, en el cual el profesor principiante se ve abocado a invertir la mayor parte de su dedicación laboral en ampliar los méritos de investigación, no contando, por lo tanto, con el tiempo suficiente para la formación y preparación adecuada de las tareas docentes. Además, el profesor principiante suele dejar a un lado la participación en la gestión de la universidad. A menudo existe, a su vez, un desconocimiento general del marco organizativo en el que el profesor principiante debe desenvolverse, a pesar de que la docencia y la investigación están influidas por la estructura y la organización existente en la universidad, y que se trata ésta de una institucion regida por órganos democráticos basada en la participación de sus integrantes.

En este artículo las autoras presentan, partiendo de su propia experiencia como profesoras principiantes, una reflexión sobre los problemas más relevantes a los que se enfrentan en sus carreras docentes. Dichas reflexiones han sido recogidas en el marco de su participación en el programa de Formación de Profesores Noveles de la Universidad de Sevilla (España). Este equipo de trabajo está constituido por profesores pertenecientes a la Escuela Superior de Ingenieros, que imparten docencia tanto teórica como práctica en diversas asignaturas de la titulación de Ingeniero de Telecomunicación. Dichos profesores principiantes han contado con la asesoría de un profesor mentor perteneciente a su misma área de conocimiento, así como con el apoyo de los profesionales del Instituto de Ciencias de la Educación de la Universidad de Sevilla. Como resultado de esta experiencia, entre las dificultades percibidas se destacan problemas en el ámbito de la enseñanza, en las relaciones interpersonales, haciendo especial referencia a la acción tutorial, y en el ámbito de gestión o contexto institucional, siguiendo la clasificación de (Sánchez-Moreno y Mayor-Ruiz, 2006). 


\section{DIFICULTADES EN EL ÁMBITO DE LA ENSEÑANZA}

La asignación de docencia al profesor novel no siempre se realiza con cierta coherencia. El sistema de asignación se suele basar en un orden de prelación en el cual los profesores noveles ocupan los últimos lugares, de forma que la elección de asignaturas se convierte, en la práctica, en una asignación. Si la docencia designada corresponde a asignaturas de primer ciclo, el profesor se enfrenta a grupos que suelen ser numerosos, con lo que los principales problemas están relacionados con el comportamiento y actitud de los alumnos en clase. Por el contrario, si la docencia asignada es de segundo ciclo, los grupos suelen ser más reducidos, pero la disciplina a impartir es más especializada. En este caso el principal problema radica en la preparación de la materia a impartir. Por otro lado, se recomienda que el profesor principiante comience su carrera docente en asignaturas de carácter práctico, y que progresivamente se vaya incorporando a asignaturas troncales u obligatorias. La realidad es que esta recomendación no siempre se puede seguir, a pesar de que facilita enormemente la inserción al profesor principiante.

\section{Planificación y organización de la asignatura}

En relación a la planificación de la docencia estipulada, el profesor principiante tiende a asimilar lo ya establecido, especialmente en la preparación del contenido de cada clase. A pesar de que este aspecto es sumamente importante, es un algo muy poco valorado por los alumnos, que sólo perciben la actuación en clase. Sin embargo, la elección de contenidos adecuados y atractivos para el estudiante fomenta la participación en clase y consigue motivar al mismo. El problema en este caso suele derivarse de la coordinación del profesor principiante con el resto de profesores de la asignatura. La gestión del tiempo en las clases es un aspecto que también exige un considerable esfuerzo por parte del docente novel; la planificación de los tiempos dedicados a diferentes tareas dentro de una clase y la preparación de contenidos adicionales para cubrir los posibles huecos que puedan aparecer, son puntos clave para el buen desarrollo de la unidad didáctica.

\section{Metodología docente}

La responsabilidad del docente no recae únicamente en la exposición de determinados contenidos, sino que es éste quien debe facilitar todo el proceso de enseñanza-aprendizaje a través de una determinada metodología. Una buena preparación del contenido y de las estrategias para dirigir el aprendizaje puede facilitar mucho la consecución de los objetivos. Es evidente la falta de formación del profesorado principiante en técnicas docentes y en general en metodologias de enseñanza, lo que puede llevar a pensar que el profesor principiante se limita a imitar las metodologías ya conocidas y tradicionales. Sin embargo, el profesor principiante suele tratar de innovar en la enseñanza, mejorar en alguna forma lo que él ha conocido.

El afán se superación, presente en el docente novel, le lleva a aprender por sí mismo e intentar utilizar otras técnicas, estrategias y recursos didácticos novedosos en el aula. A la hora de llevar a la práctica estas estrategias, el profesor se encuentra con muchas dificultades. Por un lado, la mayor complejidad de estos métodos exige un elevado tiempo de preparación de las clases. Por otro lado, existe una falta de concienciación de los alumnos en la importancia del proceso de aprendizaje. A menudo el profesor principiante se encuentra con actitudes pasivas por parte de los alumnos: limitarse a copiar y saber qué tipo de examen va a tener lugar para adquirir los conocimientos mínimos y conseguir aprobar la asignatura, (Rué, 2000). Esto le lleva a un estado de insatisfacción que le desmotiva e interfiere muy negativamente en la proactividad innata del profesor principiante. Otro aspecto a tener en cuenta es la necesidad de adaptación de la metodología a la diversidad existente entre los alumnos. A menudo se considera que las metodologías más adecuadas en cursos inferiores son aquellas que podemos denominar expositivas, mientras que en los cursos superiores, se pueden incluir estrategias de autoaprendizaje con una buena aceptación por parte del alumnado.

\section{Actualización de materiales y contenidos}

Los contenidos de las asignaturas deben ser revisados y actualizados para conseguir que los conocimientos estén en contacto con la realidad y no queden obsoletos. Esta tarea la suelen llevar 
a cabo los profesores coordinadores de las asignaturas o aquellos con más experiencia. Sin embargo, para la formación integral del profesor principiante, es conveniente participar en la elaboración y/o actualización de los contenidos de los programas de las asignaturas.

Una de las tareas en las que el profesor principiante invierte más tiempo es en la elaboración de su propio material escrito como soporte al ya existente. Esto, que a priori es altamente constructivo, puede ser perjudicial si no se comienza por evaluar lo ya existente, valorando no sólo lo defectuoso sino también lo útil y positivo. Además del material propio, el profesor principiante debe participar junto con el resto de profesores de la asignatrura en la preparación de acividades prácticas y de aplicación a la realidad.

\section{Inseguridad y falta de experiencia}

Como ya se ha comentado con anterioridad, el profesor principiante cuenta con los conocimientos científicos necesarios para dominar por completo la materia sobre la que va a impartir su clase. Esto es así dado el procedimiento de entrada en la carrera docente universitaria basado en los méritos obtenidos por el candidato. Sin embargo, la experiencia en el marco de la docencia no es un requisito indispensable. Por este motivo, el profesor principiante normalmente no se ha enfrentado nunca con un grupo de alumnos a los que transmitir sus conocimientos. Nunca ha tenido que explicarse de forma que personas no versadas en el tema que debe exponer lo entiendan, lo asimilen y, en definitiva, lo aprendan. Nunca ha tenido que contestar preguntas poco corrientes, mantener la disciplina o favorecer la motivación de su audiencia. Esta inexperiencia favorece la aparición de cierto sentimiento de inseguridad al encontrarse frente al alumnado, que afecta de forma negativa a sus propósitos. Si el alumno percibe esta inseguridad, se siente defraudado al interpretarla como falta de conocimiento y por lo tanto se fomenta su desmotivación. Esto no hace más que iniciar un círculo vicioso de incomprensión mutua ya que el docente percibe la actitud reacia de sus alumnos y entra en un estado de mayor inseguridad tal como puede apreciarse en la Fig. 1.

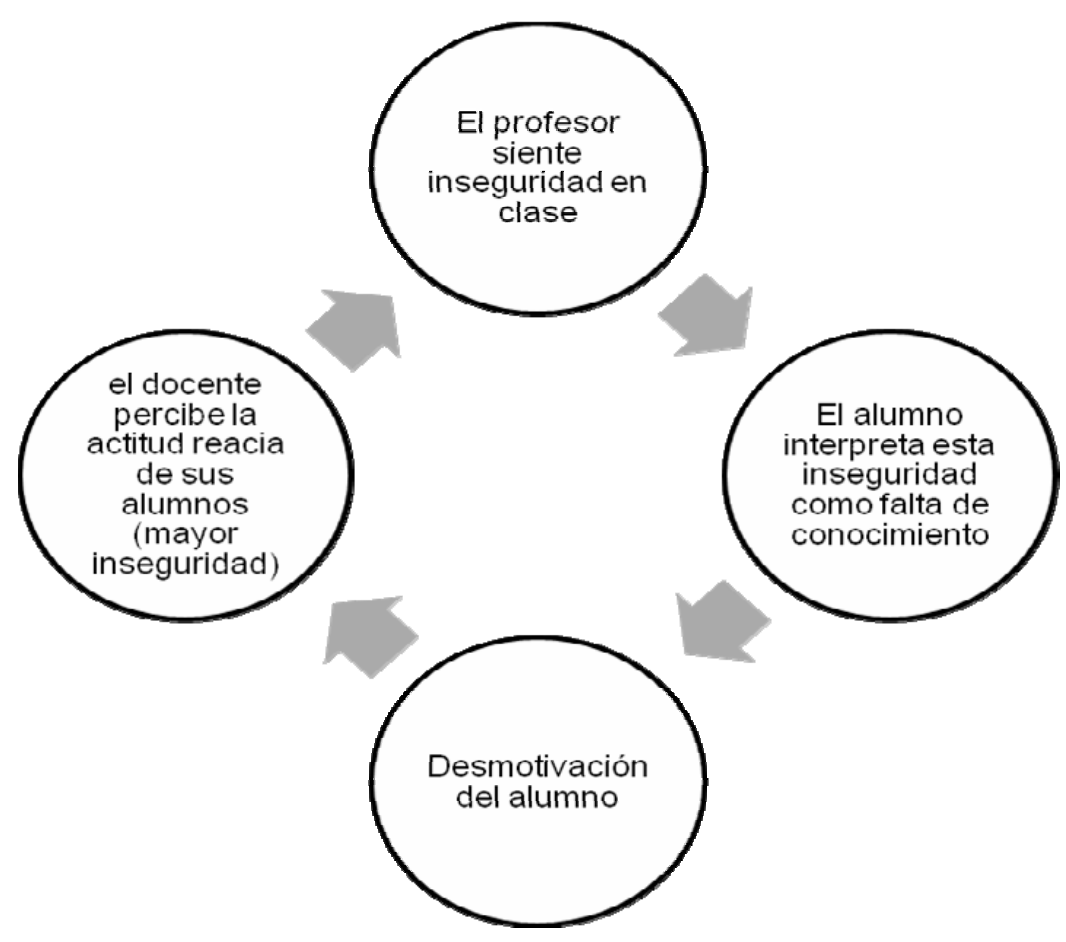

Fig. 1: La inseguridad de los profesores principiantes puede derivar en un círculo vicioso que dificulta el proceso de aprendizaje. 


\section{DIFICULTADES EN EL ÁMBITO DE LAS RELACIONES INTERPERSONALES.}

El profesorado principiante suele estar formado por personas jóvenes, de manera que no suele existir una diferencia de edad importante entre el profesor y los alumnos. A pesar de que esto debería facilitar las relaciones interpersonales, en ocasiones este hecho deriva en una dificultad por parte del profesor principiante en adoptar el papel de docente, y en una percepción por parte del alumno de esta falta de confianza, en ocasiones a partir del lenguaje no verbal del profesor. Por otro lado, la resolución de preguntas inesperadas y/o problemas surgidos en clase suele ser difícil para el profesor novel. Atender adecuadamente las preguntas inesperadas estableciendo mecanismos participativos no siempre es fácil de llevar a cabo para un profesor carente de experiencia. La actitud del profesor en el aula es crucial. Mantener una actitud calmada y sosegada utilizando un lenguaje y tono de voz adecuados es indispensable si se desea que los alumnos perciban el dominio de la materia que se expone.

\section{Eficacia de la acción tutorial}

La acción tutorial constituye una herramienta de enorme valor para la enseñanza y puede resultar complicada desde el punto de vista de un profesor principiante ya que, dada su ilusión como principiante, pretende sacarle el máximo partido a pesar de las numerosas dificultades encontradas. Es ampliamente aceptado que la tutoría es una función necesaria en todos los niveles educativos. En educación primaria y secundaria, el tiempo dedicado a las tutorías está muy bien definido aunque con matices diferentes respecto al ámbito universitario en cuanto a la función de las mismas. En la enseñanza superior la función tutorial no tiene el apoyo legal que posee en los otros niveles.

La participación en las tutorías no es obligatoria por parte de los alumnos. La mayoría de ellos apenas hace uso de ellas a lo largo del curso y es en las fechas próximas a los exámenes cuando se benefician de esta poderosa herramienta. La legislación actual en la Universidad de Sevilla obliga al profesor a reservar de tres a seis horas semanales para dedicación a tutorías, pero no obliga a llenarlas de contenido, a pesar de la importancia de las mismas en la enseñanza. La acción tutorial no sólo es beneficiosa para el alumno sino que también lo es para el propio profesor al proporcionar una retroalimentación de la que se puede nutrir el docente para conocer sus carencias. En este sentido es muy positivo el sistema de tutorías on-line, que permiten sobrellevar las limitaciones geográficas o temporales, añadiendo a la tutoría un carácter permanente. Por supuesto, estas tutorías no deben sustituir a las presénciales sino que las deben complementar. Realmente en las tutorías presenciales se consigue algo más que aclarar dudas: conocer a los alumnos y que ellos conozcan a su profesor en un contexto más libre. Además de la solución a cuestiones y problemas, existe también una función de asesoramiento académico, ampliación de fuentes bibliográficas y orientación sobre perfiles laborales, entre otras. Es el lado más humano y amable de las tutorías. La tutoría colegiada, en la que un grupo de profesores hace un seguimiento de los alumnos a partir de grupos constituidos, es otra modalidad muy interesante que podría beneficiar al profesor principiante ya que existe un beneficio para el alumno y un beneficio para un profesor novel que puede aprender de la experiencia de otros profesores.

\section{DIFICULTADES EN EL ÁMBITO DE LA GESTIÓN}

Tradicionalmente, en la Universidad siempre se le ha concedido más importancia a la función investigadora descuidando la docente, sintiendo que un esfuerzo en la mejora de esta última no es valorado por la institución. Uno de los pricipales problemas para el profesor universitario consiste en la necesidad de un balance entre las tareas docente, investigadora y de gestión. El sistema organizativo de la Universidad es bastante desconocido para el profesor principiante. Podríamos hablar de miopía colectiva (Wong, 2005) en el sentido de la dificultad de apreciación de las características de la Universidad así como de la existencia de mecanismos que obstaculizan la realización de cambios de verdadero calado en su estructura. Sería necesaria una visión de la misma que, desde cierta distancia, permita la objetividad y la percepción de la realidad. 
Al no conocer el sistema organizativo de la Universidad, el docente principiante se limita a trabajar dentro de una estructura ya establecida, siguiendo unas pautas predeterminadas que supone persiguen el bien común. Al inicio de la carrera dentro de la Universidad no se recibe información sobre el funcionamiento de la misma y tan sólo se aprende cuando surge algún problema que atañe personalmente al profesor o a su entorno más cercano. Un mayor conocimiento del sistema organizativo de la Universidad puede ayudar también a nivel de gestión, una de las funciones del profesor dentro de la Universidad. En este aspecto, el conocimiento de la organización de la Universidad debería facilitar el desempeño de su trabajo al profesorado, pero el grado en el que el docente principiante se encuentra capacitado para implicarse en la organización de la Universidad puede ser discutido. El hecho de que el docente deba dividir su tiempo entre las tres funciones (docente, investigadora y gestión), hace que dedicar parte del mismo a conocer y participar en el funcionamiento de la Universidad represente un esfuerzo no recompensado por la propia institución.

\section{Conflicto Actividad Investigadora - Carga Docente}

Como se ha expuesto con anterioridad, el trabajo del docente universitario no sólo se limita a la impartición de las clases sino que el profesor debe llevar a cabo una actividad investigadora de relevancia. Esta actividad es crucial para su futuro, ya que para continuar en la carrera docente universitaria se exige un determinado nivel científico valorado mediante el número de artículos, revistas o capítulos de libros elaborados, participación en congresos y otra serie de actividades no docentes. La actividad puramente educadora no siempre tiene un peso importante en dichas evaluaciones con lo que podemos llegar a un conflicto entre estas dos facetas.

En un marco ideal en el que el profesor tuviera poca carga docente con un número de alumnos reducido, este problema no se daría. La compaginación de ambas facetas sería algo natural y altamente positivo ya que el alumno se beneficiaría de los conocimientos cada vez más profundos del profesor sobre su materia. Sin embargo, la realidad es radicalmente opuesta, especialmente para el profesor principiante. Es muy usual que al profesor principiante se le asignen unas materias que no son del ámbito de su investigación. Al carecer de experiencia, éste dedicará gran parte de su tiempo al estudio de la materia que debe tratar, así como a la elaboración de nuevo material, estructuración de contenidos, etc. Esto va en contraposición con el tiempo que necesita dedicar a la investigación, reduciéndose enormemente. Puede que incluso la preparación pedagógica y docente quede relegada a un segundo término con el consiguiente perjuicio para los alumnos. Por otro lado no debemos olvidar las tareas de carácter burocrático y de gestión que también consumen una parte significativa de la jornada laboral.

\section{Espacio Europeo de Educación Superior}

En relación con la reestructuración del modelo universitario en el nuevo "Espacio Europeo de Educación Superior (EEES)" (Ministerio de Educación, 2003), los profesores principiantes en la actualidad nos encontramos con un reto mucho mayor. A todo lo expuesto anteriormente hay que añadir el relativo momento de desorden y desconcierto provocado por la reforma. Es bien sabido que dentro del EEES se propone un nuevo modelo de enseñanza centrado en el aprendizaje y basado en una serie de competencias que hacen al estudiante protagonista activo de su aprendizaje. Este modelo de enseñanza conlleva una revisión de la metodología docente actual y además es necesario un profundo cambio en las infraestructuras.

El modelo andragógico de enseñanza, (Cabrera, 2003), posee muchas de las características necesarias para ser un modelo viable dentro del EEES. No obstante, hoy en día, tal y como está estructurada la docencia universitaria, hay materias en las que el modelo andragógico de enseñanza que nos permite pensar en las necesidades específicas del aprendizaje adulto, es difícilmente aplicable. En asignaturas con un contenido denso y un elevado número de créditos, el modelo de enseñanza andragógico es inviable, y las clases magistrales se presentan como una alternativa posible. Los límites, pues, no están marcados, sino que pueden y se deben utilizar diferentes metodologías. 
Al día de hoy, las asignaturas están en proceso de adaptación, y eso influye mucho en el tiempo que un profesor tiene que dedicar a realizar dicha adaptación, máxime cuando se trata de un profesor principiante. Si bien a un profesor principiante le es difícil participar en la elección de las metodologías docentes que se aplican a las asignaturas, sí realizan otros tipos de tareas. Por ejemplo, la actualización del material docente a nuevos formatos que ayuden a la adaptación al nuevo entorno formativo, la preparación de las clases en su nuevo formato, etc. Esto supone una carga adicional de difícil solución, si no se presta atención a las necesidades especiales del docente universitario frente a la reforma.

\section{CONCLUSIONES}

La participación de las autoras de este artículo en un programa de Formación de Profesores Noveles ha dado lugar a una reflexión sobre los problemas más relevantes a los que se enfrentan los profesores principiantes en el marco universitario. Las dificultades expuestas se han clasificado en tres ámbitos: el de la enseñanza, el de las relaciones interpersonales y el de la gestión. Del análisis y estudio presentado se pueden deducir las siguientes conclusiones principales:

i) La actitud con la que el profesor novel afronta por primera vez su actividad docente en la Universidad es, por norma general, de interés y motivación.

ii) Los inconvenientes a los que se enfrenta suelen derivarse de su falta de formación pedagógica y experiencia docente. La inseguridad que ello le produce obstaculiza su capacidad de comunicación y de transmisión de conocimientos.

iii) Sus recursos se ven limitados por el desconocimiento de las mejores técnicas docentes que le permitan motivar al alumno y mantener su atención.

iv) La planificación de los contenidos y actividades de las clases, la gestión del tiempo, la resolución de situaciones inesperadas en clase o la actividad tutorial son facetas en las que parece ser necesario incrementar los esfuerzos en la formación de los docentes.

v) El desconocimiento de la organización universitaria imposibilita al docente el cambio o mejora dentro de la misma. De esta forma, la actividad de gestión y administrativa puede ser demasiado absorbente para aquél que no conoce los mecanismos adecuados.

vi) El conflicto investigación-docencia es uno de los mayores problemas con los que se encuentra el profesor, ya que siente que el tiempo dedicado a la mejora de su actividad docente no es adecuadamente valorado por la institución.

vii) Por último, los retos ante la reforma del modelo universitario español en el nuevo Espacio Europeo de Educación Superior suponen un trabajo adicional para todos los docentes universitarios dado que hay que adecuar la enseñanza a otros métodos pedagógicos.

viii) Una de las principales cualidades del profesor principiante a la hora de sobreponerse a las dificultades expuestas es, en opinión de las autoras, su afán de superación, que le lleva a aprender por sí mismo e intentar mejorar la forma de transmitir sus conocimientos de la materia con una gran capacidad de innovación.

\section{AGRADECIMIENTOS}

Las autoras expresan su agradecimiento al Profesor Dr. D. Justo Calvo Aguilar por sus valiosos consejos y su labor como mentor.

\section{REFERENCIAS}

Bozu, Z., El profesorado universitario novel y su proceso de inducción profesional, Magis, Revista Internacional de Investigación en Educación, 2, pp 317-328, (2009) 
Cabrera, J. A. (Universidad de La Habana "Fructuoso Rodríguez. Pérez"), Andragogía: ¿Disciplina necesaria para la formación de directivos?, (en línea), 2003.

http://www.monografias.com/trabajos14/andragog/andragog.shtml. Acceso 22 de Septiembre (2008).

Colén, M., Cano, E., Lleixà, T y Medina, J. L., Las necesiades formativas del profesorado universitario novel para el ejercicio de la función docente, 1er Congreso Internacional Docencia Universitaria e Innovación, Universidad de Barcelona, Autónoma y Politécnica, (2000)

Cruz, M. A. de la, Gómez E. y Martínez, M. F. El programa de formación inicial para la docencia universitaria en la Universidad Autónoma de Madrid, (en línea), 1999.

http://www.ub.es/forum/Conferencias/sadu.htm, Acceso 22 de Septiembre (2008)

Dunkin, M., The induction of academic staff to a University: process and product, Higher Education, 20, 1, pp. 47-66, (1990)

Howwey, K., Mentor-teachers as inquiring professionals, theory into practice, Mentoring teachers, 27 (3), pp. 210-213, (1988)

Marcelo, C. Estudios sobre estrategias de inserción profesional en Europa, Revista Iberoamericana de Educación, 19, pp 101-144, (1999)

Mignorance-Díaz, P., Mayor-Ruíz, C. y Marcelo-García, C., El primer año en la universidad. Análisis de problemas de profesores principiante, Reu: Revista de Enseñanza Universitaria, 5, pp.19-36, (1993)

Ministerio de Educación, Cultura y Deporte, La integracion del sistema universitario español en el espacio europeo de enseñanza superior. Documento Marco (2003), http://web.micinn.es/05_Investigacion/032CoopIntern/00@OfEuropea/012EEES/04@SpE/00Dtos/Documento_Marco.pdf. Acceso: 22 de Septiembre 2008

Rué, D., Aprendizaje activo. Alternativas a la lección magistral, Congreso Internacional de Docencia Universitaria e Innovación, Barcelona, España, Junio (2000),

http://dewey.uab.es/paplicada/htm/papers/Paper_aprendizajeactivo_rue.htm. Acceso: 22 de Septiembre 2008.

Sánchez-Moreno, M. y Mayor-Ruiz, C., Los jóvenes profesores universitarios y su formación pedagógica. Claves y controversias. Revista de Educación, 339, pp. 923-946, (2006)

Veenam, S., Perceived Problems of Beginning Teachers, Review of Educational Research, 54, (2), pp. 143-178, (1984)

Wong, M., Organizational Learning via Expatriate Managers: Collective Myopia as Blocking Mechanism. Organization Studies: 26(3), pp 325-350, (2005) 